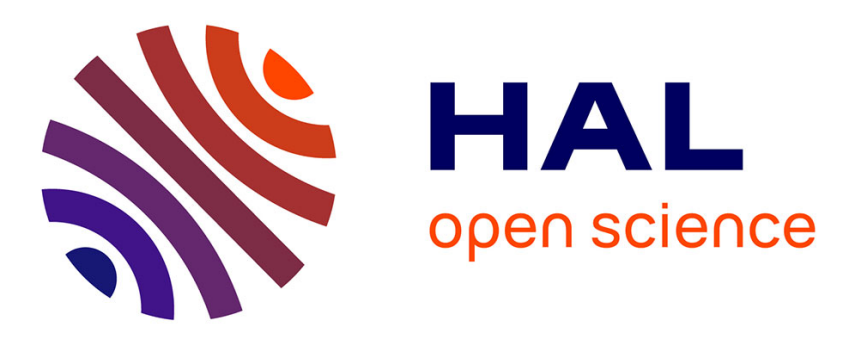

\title{
Sur certaines propriétés des alliages supraconducteurs non magnétiques
}

C. Caroli, P.-G. de Gennes, J. Matricon

\section{To cite this version:}

C. Caroli, P.-G. de Gennes, J. Matricon. Sur certaines propriétés des alliages supraconducteurs non magnétiques. J. Phys. Radium, 1962, 23 (10), pp.707-716. 10.1051/jphysrad:019620023010070700 . jpa-00236667

\section{HAL Id: jpa-00236667 https://hal.science/jpa-00236667}

Submitted on 1 Jan 1962

HAL is a multi-disciplinary open access archive for the deposit and dissemination of scientific research documents, whether they are published or not. The documents may come from teaching and research institutions in France or abroad, or from public or private research centers.
L'archive ouverte pluridisciplinaire HAL, est destinée au dépôt et à la diffusion de documents scientifiques de niveau recherche, publiés ou non, émanant des établissements d'enseignement et de recherche français ou étrangers, des laboratoires publics ou privés. 


\title{
SUR CERTAINES PROPRIÉTÉS DES ALLIAGES SUPRACONDUCTEURS NON MAGNETIQUES
}

\author{
Par C. CAROLI, P. G, DE GENNES et J. MATRICON. \\ Laboratoire de Physique des Solides, Faculté des Sciences, Orsay.
}

\begin{abstract}
Résumé. - On étudie le rôle de la diffusion des électrons par les impuretés sur la température de transition des alliages supraconducteurs non magnétiques : 1) Aux très faibles concentrations en impuretés, l'effet principal est lié à la disparition de l'anisotropie du " gap ». On calcule sur un modèle simple la forme détaillée du diagramme température de transition/concentration dans cette région ; 2) Dans le domaine des alliages "sales ", la première approximation, examinée par Anderson, revient à supposer que le paramètre d'ordre $\Delta(\boldsymbol{r})$ est le même en tous points de l'alliage. Nous montrons ici comment l'on peut tenir compte de la distorsion locale de $\Delta(r)$ au voisinage de chaque impureté par une méthode d'itération. Les corrections résultantes sur la température de transition sont importantes lorsque les valences des deux constituants sont très différentes. La distorsion de $\Delta(r)$ au voisinage de la surface d'un supraconducteur pur peut être calculée par la même méthode. Ceci permet de préciser les conditions aux limites qui doivent être composées sur une telle surface dans les équations de Landau-Ginsburg.
\end{abstract}

\begin{abstract}
Some effects of electron scattering by impurities in superconducting lalloys are calculated : 1) At very small impurity concentrations, the major phenomenon is the destruction of the anisotropy in the energy gap. This is computed in detail for a simple model ; 2) In the " dirty superconductor" range the first approximation, introduced by Anderson, amounts to replace the " order parameter" $\Delta(\boldsymbol{r})$ by its space average. We show that local variations of $\Delta(\boldsymbol{r})$ can be taken into account by an iteration process; they are important for impurities which are very different in charge from the matrix. They can also be studied in the vicinity of the free surface of a pure superconductor : this gives a better understanding of the boundary conditions to be applied at the Landau-Ginsburg equations.
\end{abstract}

I. Introduction. - Les principaux effets d'impuretés non magnétiques sur la température de transition d'un alliage supraconducteur sont liés à :

a) La destruction de l'anisotropie du "gap " accompagnée d'une baisse de la température de transition. Dans les supraconducteurs doux cet effet prend place à de très faibles concentrations en impuretés, et il est alors clairement visible sur les diagrammes expérimentaux donnant la température de transition $T_{c}$ en fonction de la concentration [1]. Pour une même matrice on constate que $T_{\text {s }}$ aux très faibles concentrations dépend uniquement de la résistivité résiduelle introduite par les impuretés (ou par des défauts de réseau), et plus précisément du rapport entre le libre parcours moyen $l$ et la longueur de cohérence $\xi_{0}$ du métal pur au zéro absolu [2]. Le principe de l'explication de ces effets a été donné par Anderson [3]. Nous les calculons ici quantitativement (section II) sur un modèle très simplifié.

b) Une modification de la densité de niveaux et de l'interaction électron-électron. Ces effets ont été examinés en premier par Abrahams et Weiss [4]. Les résultats expérimentaux, notamment dans les séries de transition, suggèrent que la densité de niveaux est en fait le facteur essentiel. Toutefois la description macroscopique de l'effet des impuretés par l'intermédiaire de cette densité n'est qu'une première approximation : il faut tenir compte plus en détail de la distorsion des fonctions d'onde par les impuretés. Ceci a été discuté par Suhl [5] et est calculé en détail par une autre méthode dans la section III.

D'une façon générale, nous ignorons complètement dans cet article les effets dûs à une modification de l'interaction électron-électron par les impuretés. Nous nous intéresserons uniquement à l'effet des impuretés sur les fonctions d'onde à un électron dans le métal normal et à ses conséquences dans l'état supraconducteur. Les corrections (en géné 'al relativement faibles) dues à une modification de l'interaction seront discutées dans un article ultérieur.

II. Alliages très dilués. - Nous abordons ici le cas d'un supraconducteur doux, où la longueur de cohérence $\xi_{0}$ au zéro absolu est élevée $\left(\sim_{10^{-4}} \mathrm{~cm}\right)$ dans lequel on introduit une très faible concentration d'impuretés, de façon à ce que le libre parcours moyen $l$ soit comparable à $\xi_{0}$.

Dans le métal pur le seuil d'énergie, ou gap $\Delta_{p}$, pour une paire $(p,-p)$ dépend de la position de $p$ sur la surface de Fermi, à cause des anısotropies de cette surface et de l'interaction électron-électron. Lorsque l'on ajoute des impuretés, chaque paire $(p,-p)$ n'est plus pure mais contient des composantes $\left(p^{\prime},-p^{\prime}\right)$ à cause des effets de diffusion. Le gap $\Delta_{p}$ tend alors à prendre une valeur uniforme sur toute la surface de Fermi. C'est cet effet que nous allons calculer maintenant. 
1. Approximations. - Les principales approximations faites cont les suivantes :

a) L'interaction attractive entre électrons est décrite comme une interaction statique non locale.

$$
a_{p_{1} \alpha}^{+} a_{p_{3} \beta}^{+} a_{p_{3} \beta} a_{p_{6}^{\alpha}} \quad V\left(p_{1} p_{2} p_{3} p_{4}\right)
$$

(où les $a^{+}$sont des opérateurs de création dans les états de Bloch du métal) et nous négligeons l'influence des impuretés sur $V$. $\alpha$ et $\beta$ sont des indices de spin.

b) Nous négligeons le changement de la densité de niveaux dû aux impuretés. Les erreurs introduites par cette approximation sont faibles pour les alliages très dilués qui nous intéressent ici, et donnent des effets linéaires aux faibles concentrations, donc relativement faciles à soustraire.

c) Par contre nous tenons compte de la diffusion des électrons par les impuretés qui joue ici le rôle essentiel, mais nous simplifions toutefois la situation en supposant que la probabilité de transition d'un état $k$ vers un autre état $k^{\prime}$ est la même quel que soit le couple d'états considérés $k, k^{\prime}$ (au voisinage de la surface de Fermi).

d) Pour pouvoir associer aux états $p$ un gap bien défini $\Delta_{p}$, nous devons faire une simplification un peu analogue à l'approximation des bandes rigides, familière dans l'étude des alliages de métaux normaux.

Une discussion détaillée de cette approximation sera faite dans la section IV où l'on étudie la variation de $\Delta$ dans une représentation spatiale.

e) Pour pouvoir déterminer explicitement la forme du gap $\Delta_{p}$, il est commode de simplifier la structure de l'interaction $V_{p q}=V(p,-p, q,-q)$ qui couple les paires $(p,-p),(q,-q)$. Nous supposerons que $V_{p q}$ dépend de la direction de $p$ et $q$, mais que $V_{p q}$ est pratiquement indépendant des énergies $\xi_{p}, \xi_{q}$ des etats $p$ et $q$, comprises dans un intervalle d'épaisseur $2 \omega_{\mathbf{D}} \hbar$, autour de l'énergie de Fermi $E_{\mathrm{F}}$. Hors de cette région on prend $V_{p q}=0$. Cette approximation 'est analogue à celle introduite initialement par B. C. S. [6] mais ici $V_{p q}$ dépend des directions $p$ et $q$, donc les effets d'anisotropie ne sont pas éliminés.

L'approximation (e) a deux défauts :

1) Allors que l'interaction réelle dépend surtout de la différence des énergies $\xi_{p}$ et $\xi_{q}$, l'interaction substituée dépend séparément de $\xi_{p}$ et $\xi_{q}$. Ceci peut introduire des effets parasites lorsque la fréquence de collision $1 / \tau$ due aux impuretés devient comparable à $\omega_{\mathrm{D}}$. Toutefois nous n'étudions ici que le domaine où $h / \tau \sim k_{\mathrm{B}} T_{0}$ où $T_{0}$ est le point de transition du métal pur, et nous supposons $k_{\mathbf{B}} T_{0} \ll \hbar \omega_{\mathrm{D}}$ (limite de couplage faible). Donc l'inconvénient cité n'est pas grave.

2) En toute rigueur, la fréquence de coupure $\omega_{\mathbf{D}}$ est la fréquence du phonon de vecteur d'onde $p-q$ : elle dépend donc de $p$ et $q$, et en particulier elle est faible lorsque $p$ est voisin de $q$.
Mais l'élément de matrice $V_{p q}$ est faible dans ce cas, et $\omega_{D}$ n'intervient de toute façon qu'à l'intérieur d'un logarithme. Il est donc raisonnable de prendre $\omega_{D}$ constanten première approximation.

2. ÉQuation Du GaP. - Le calcul se conduit de la façon suivante : définissons un "gap "

$$
\Delta\left(p_{1} p_{2}\right)=\sum_{p_{3} p_{4}} V\left(p_{1}, p_{2}, p_{3}, p_{4}\right)<a_{p_{3} \downarrow} a_{p_{4} \uparrow}>\text {. }
$$

$\mathrm{Au}$ voisinage de la température de transition, $\Delta$ obéit à une équation de self consistence linéaire, dérivée en premier lieu par Gorkov [7]

$$
\begin{aligned}
& \Delta\left(p_{1} p_{2}\right) \\
& =\sum_{p_{3} p_{6} p_{5} p_{6}} V(1234) T \sum_{\omega} G_{\omega}\left(p_{5} p_{3}\right) G_{-\omega}\left(p_{6} p_{4}\right) \Delta\left(p_{5} p_{6}\right)
\end{aligned}
$$

Les $G$ sont des fonctions de Green à un électron dans le métal normal en présence des impuretés. Si l'on introduit les fonctions d'onde exactes à un électron $w_{n}(p)$ (dans une représentation d'impulsion), on peut écrire explicitement les $G$ sous la forme

$$
G_{\omega}\left(p_{1} p_{2}\right)=\sum_{n} \frac{1}{l \omega-\xi_{n}} w_{n}^{*}\left(p_{1}\right) w_{n}\left(p_{2}\right) .
$$

La somme $\sum_{\omega}$ dans (II.2) est étendue aux valeurs discrètes de la fréquence $\omega=2 \pi\left(k+\frac{1}{2}\right) T$ ( $k$ entier algébrique; $T:$ température, on prend $\hbar$ et la constante de Boltzmann égaux à 1) $\xi_{n}$ est l'énergie à l'état $n$ comptée à partir du niveau de Fermi.

Dans l'équation (II.2) les $G$ et les $\Delta$, dépendent d'une façon complexe de la position géométrique de toutes les impuretés dans la matrice. Nous allons maintenant faire une moyenne sur toutes les configurations possibles de ces impuretés. On obtient dans le nombre de droite une expression du type $\overline{G_{\omega} G_{-\omega} \Delta}$, que nous remplacerons par le produit $\overline{G_{\omega} G_{-\omega}} \bar{\Delta}$. C'est cette substitution qui correspond à l'approximation citée plus haut, nous verrons plus tard (section IV) qu'elle est justifiée pour des impuretés dont la valence ne diffère pas trop de celle de la matrice.

(Par contre, nous ne ferons pas la substitution $\overline{G_{\omega} G-\omega} \rightarrow \overline{G_{\omega}} \overline{G_{-\omega}}$ qui serait tout à fait injustıtiée aans le cas present.) En raison de l'invariance de translation, les seules valeurs moyennes non nulles pour le gap sont alors de la forme

$$
\overline{\Delta(p,-p)}=\Delta_{p}
$$

et l'équation (II.2) se réduit à :

$$
\Delta p=T \sum_{\omega} \sum_{p^{\prime}} V_{p p^{\prime}} S_{\omega}\left(p^{\prime} q\right) \Delta q
$$

avec

$$
\begin{aligned}
V p p^{\prime} & =V\left(p,-p, p^{\prime},-p^{\prime}\right) \\
S_{\omega}(p q) & =\frac{G_{\omega}(p q) G_{-\omega}(-q-p) .}{}
\end{aligned}
$$


La température $T$ pour laquelle le système linéaire (II.4) admet une solution non triviale est la température de transition de l'alliage.

3. Calcul des moyennes. - Les moyennes $\overline{G_{\omega}}$ et $S_{\omega}(p q)$ peuvent être calculées pour les alliages dilués, par des méthodes qui sont maintenant classiques [8], On obtient :

$$
\begin{gathered}
G_{-\omega}(-p,-q) \overline{G_{\omega}(p q)}=\delta p q \frac{1}{\xi_{p}^{2}+\eta^{2} \omega^{2}} \\
\eta=1+\frac{1}{2 \tau p|\omega|}
\end{gathered}
$$

$\tau_{p}$ est la durée de vie de l'état $(p)$, que nous supposons pour simplifier indépendant de $p$. En ce qui concerne $S_{\omega}(p q)$, il est obtenu, à l'approximation de Born, à partir de l'équation intégrale [8]

$$
\begin{aligned}
S_{\omega}(p q)=\overline{G_{\omega}(p p)} \overline{G_{-\omega}(-p-p)} \\
\\
\quad\left[\delta p q+\sum_{f} n\left|W_{p^{\prime} f}\right|^{2} S_{\omega}(f, q)\right] .
\end{aligned}
$$

Le terme diagonal représente la propagation d'une paire d'électrons dans les états $p$ et $-p$; le deuxième terme représente l'amplitude de diffusion de cette paire vers les états $(f,-f)$. $W_{\text {Pf }}$ est l'élément de matrice du potentiel dû à une impureté (il figure au carré puisque deux électrons sont diffusés simultanément) ; $n$ est le nombre d'impuretés par $\mathrm{cm}^{3}$. Le temps de relaxation $\tau_{p}$ est relié à ces quantités par la relation

$$
\frac{1}{\tau_{p}}=2 \pi n \sum_{f}\left|W_{p f}\right|^{2} \delta\left(\xi_{f}-\xi_{p}\right)
$$

L'équation (II.7) est une équation intégrale assez malaisée à résoudre dans un métal anisotrope. On la simplifie en remplaçant $\left|W_{p f}\right|^{2}$ par une constante $W^{2}$; dans ce cas la somme $\sum$ donne un résultat indépendant de $p$, et l'on obtient aisément à partir de (II.6), (II.7) et (II.8)

$$
S_{\omega}(p q)=\frac{1}{\xi_{p}^{2}+\eta^{2} \omega^{2}}\left[\delta p q+\frac{\eta}{2 \pi N \tau} \frac{1}{\xi_{q}^{2}+\eta^{2} \omega^{2}}\right]
$$

$N$ est la densité totale de niveaux à l'énergie de Fermi $E_{\mathrm{F}}$ et $\xi_{\mathrm{p}}=p^{2} / 2 m-E_{\mathrm{F}}$.

4. RÉduction de L'ÉQuation intÉGRale. Nous pouvons maintenant retourner à l'équation intégrale (II.4) dont tous les termes sont explicités, et chercher sa solution. On peut commencer par intégrer par rapport aux énergies $\xi_{p}$ et $\xi_{q}$. pour des directions fixées de $p, q, q^{\prime}$. Dans la limite $d u$ couplage faible que nous étudions, le gap $\Delta q$ est pratiquement indépendant de l'énergie au voisinage de la surface de Fermi. On obtient ainsi

$$
\Delta_{p}=\int \mathrm{d} \sigma_{q} N_{q} \Delta q\left[A V_{p q}+\frac{B}{N} \int \mathrm{d} \sigma_{p^{\prime}} N_{p^{\prime}} V_{p p^{\prime}}\right]
$$

où d $\sigma_{q}$ est l'élément d'aire de la surface de Fermi, $N_{q} \mathrm{~d} \sigma_{\downarrow}$ la densité correspondante de niveaux par unité d'énergie, et

$$
\begin{aligned}
& A=T \sum_{\omega} \int_{-\omega_{D}}^{\omega_{D}} \frac{d \xi}{\xi^{2}+\eta^{2} \omega^{2}} \\
&=T \sum_{\omega} \frac{2}{\eta|\omega|} \operatorname{Arctg} \frac{\omega_{D}}{\eta|\omega|} \\
& B=T \sum_{\omega} \int_{-\omega_{D}}^{\omega_{D}} \frac{d \xi}{\xi^{\prime}+\eta^{2} \omega^{2}} \\
& \qquad \int_{-\infty}^{\infty} N d \xi^{\prime} \frac{\eta}{\xi^{\prime 2}+\eta^{2} \omega^{2}} \frac{1}{2 \pi N \tau} \\
&=T \sum_{\omega} \frac{1}{\eta|\omega|^{2} \tau} \operatorname{Arctg} \frac{\omega_{D}}{\eta|\omega|}
\end{aligned}
$$

Les sommes $\sum$ qui figurent dans (II.11) et (II.12) divergeraient logarithmiquement si Arc $\operatorname{tg} \omega_{\mathrm{D}} / \eta \omega$ restait constamment égal à $\pi / 2$.

En fait, la présence de l'Arc tg introduit une coupure pour $\eta|\omega| \sim \omega_{\mathrm{D}}$. Dans le domaine qui nous intéresse $\left(1 / \tau \sim T_{0} \ll \omega_{D}\right)$ la position de la coupure n'est pratiquement pas modifiée par la présence du facteur $\eta$ : On peut remplacer $\eta$ par 1 dans les Arc tg ( ). Il ne serait d'ailleurs même pas correct de garder $\eta$ à cet endroit, car les corrections correspondantes viennent pour une grande part des approximations faites, $(a)$ et $(e)$, et n'ont pas de signification physique). Par contre, il est important de garder la valeur exacte de $\eta$ dans les autres facteurs de (II.11) et (II.12). Ici, en effet, $\eta$ intervient d'une façon non logarithmique. On écrira donc :

$$
\begin{gathered}
A=2 T \sum_{\omega} \frac{1}{\eta|\omega|} \operatorname{Arctg} \frac{\omega_{\mathrm{D}}}{\omega} \\
B=T \sum_{\omega} \frac{1}{\eta|\omega| \tau} \operatorname{Arctg} \frac{\omega_{\mathrm{D}}}{\omega} .
\end{gathered}
$$

On remarque que :

$$
K=A+B=2 T \sum_{\omega} \frac{1}{|\omega|} \operatorname{Arctg} \frac{\omega_{\mathrm{D}}}{\omega} .
$$

Cette expression est celle qui figure dans le calcul habituel de la température de transition dans la méthode B. C. S, et elle peut être mise sous la forme

$$
K=\log \frac{2 \mathrm{e} \gamma}{\pi} \frac{\omega_{\mathrm{D}}}{T}
$$

où $\gamma=1+1 / 2+\ldots+1 / n-\log n$ est la constante d'Euler. Par des méthodes analogues on peut exprimer $B$ en termes de la fonction polygamma $\psi_{1}(x)=\Gamma^{\prime}(x) / \Gamma(x)$

$$
\begin{aligned}
& B=\psi_{1} \frac{(U+1)}{2}-\psi_{1}\left(\frac{1}{2}\right) \\
& U=\frac{1}{2 \pi \tau T} \cdot
\end{aligned}
$$


Dans le matériau pur $B$ est nul et $A=R$. Dans le matériau très impur, $B$ devient voisin de $K$ et $A$ devient petit. (Toutefois ces formules ne sont valables que pour $\left.1 / \tau \ll \omega_{D}\right)$. On a finalement ramené l'équation intégrale tridimensionnelle (II.4) à une équation réduite (II.10) portant uniquement sur la surface de Fermi.

5. Solution par itération. - Pour résoudre approximativement (II.10) nous nous restreignons au cas où l'anisotropie de la surface de Fermi dans le métal pur est relativement faible. L'approximation d'ordre zéro correspond alors à un gap constant $\Delta_{\mathrm{p}}^{\mathbf{0}}=\Delta^{\mathbf{0}}$. Effectuons une première itération à partir de cette valeur : nous obtenons d'après (II.10)

$$
\Delta_{p}^{(1)}=\frac{K}{N} F_{p} \Delta^{0}
$$

avec

$$
F p=d \sigma p^{\prime} V_{p p^{\prime}} N
$$

La deuxième itération donne :

$$
\frac{\Delta_{p}^{(2)}}{\Delta^{(0)}}=\frac{K}{N} \int \mathrm{d} \sigma_{q} N_{q} F_{q}\left(A V_{p q}+\frac{B}{N} F_{p}\right)
$$

Nous obtiendrons une équation approchée porr la température de transition en écrivant que les valeurs moyennes de $\Delta_{\mathfrak{p}}^{1}$ et $\Delta_{\mathfrak{p}}^{2}$ sur la surface c'e Fermi sont égales. On trouve, avec

$$
\begin{gathered}
U=\int \mathrm{d} \sigma_{p^{\prime}} N_{p^{\prime}} F_{P^{\prime}} \\
<\Delta^{(1)}>=\int \Delta_{p}^{1} N_{p} \mathrm{~d} \sigma p=K U \Delta^{0} \\
<\Delta^{(2)}>=\int \Delta_{p}^{2} N_{p} \mathrm{~d} \sigma \bar{p}=K\left(A I+B U^{2} I N\right) \Delta^{0}
\end{gathered}
$$

avec

$$
I=\int \mathrm{d} \sigma p N_{p} F_{\mu}^{2}
$$

La condition obtenue en égalant (II.20) et (II.21) s'écrit

$$
U \mid I=K-\lambda B .
$$

La constante sans dimensions

$$
\lambda=\frac{I N-U^{2}}{I N}=\frac{\left.\left\langle F_{\nu}^{2}\right\rangle-<F_{p}\right\rangle^{2}}{\left\langle F_{\nu}^{2}\right\rangle}
$$

mesure l'importance des effets d'anisotropie. Elle est toujours positive.

6. Discussion. - Regroupons nos formules (II.23), (II.14) et (II.15). La température de transition $T_{0}$ de l'alliage est donnée en fonction de la température de transition $T_{0}$ du métal pur, et ciu paramètre $U=\hbar / 2 \pi \tau k_{B} T_{\mathrm{c}}$ par l'équation :

$$
T_{\mathrm{c}}=T_{\mathrm{c}} \mathrm{e}^{\left.\left.-\lambda \psi_{1}\right]\left(1+\frac{\sigma}{2}\right)-\psi_{1}\left(\frac{1}{2}\right)\right]}
$$

$\lambda$ est toujours positif, et $T_{\mathrm{c}}<T_{0}$, conformément aux prévisions d'Anderson [3]. La formule (II.25) n'est valable que pour $\lambda$ petit, c'est-à-dire pour un matériau faiblement anisotrope (cette condition assure la convergence de notre méthode d'itération). Elle suppose en outre $\omega_{D} \tau \ll \mid$. Dans ces conditions on peut d'ailleurs simplifier (II.25) et écrire :

$$
\begin{gathered}
\frac{T_{\mathrm{c}}}{T_{0}}=1-\lambda\left[\psi_{1}\left(\frac{1}{2}\right)+\frac{U_{0}}{2}-\psi_{1}\left(\frac{1}{2}\right)\right] \\
U_{0}=\frac{2 \pi \tau k_{B} T_{0}}{\hbar}=0,88 \frac{\xi_{0}}{l}
\end{gathered}
$$

où $\xi_{0}=0,18 \frac{\hbar v_{\mathrm{F}}}{k_{\mathrm{B}} T_{\mathbf{0}}}$ est la longueur de cohérence du matériau pur au 0 absolu, et $l=v_{\mathrm{F}} \tau$ le libre parcours moyen ( $v_{\mathrm{F}}$ étant la vitesse des électrons au niveau de Fermi).

La courbe donnant $\left(T_{\mathrm{c}}-T_{0}\right) / \lambda T_{0}$ en fonction de $\xi_{0} / l$ est représentée sur la figure. Pour la comparer aux résultats expérimentaux, il faut soustraire de ceux-ci un terme linéaire en concentration représentant l'effet plus (faible) des changements de la densité de niveaux (et de l'interaction électron-électron) sur la température de transition.

Cette soustraction est délicate, à cause de la forme logarithmique de (II. $25^{\prime}$ ) pour $\xi_{0} / l>1$ :

$$
\frac{T_{\mathrm{c}}}{T_{0}} \sim 1-\lambda \log U_{0}\left(\text { pour } U_{0}>1\right)
$$

Il est donc particulièrement intéressant de considérer des impuretés pour lesquelles les effets "lents" sont faibles. C'est le cas des impuretés

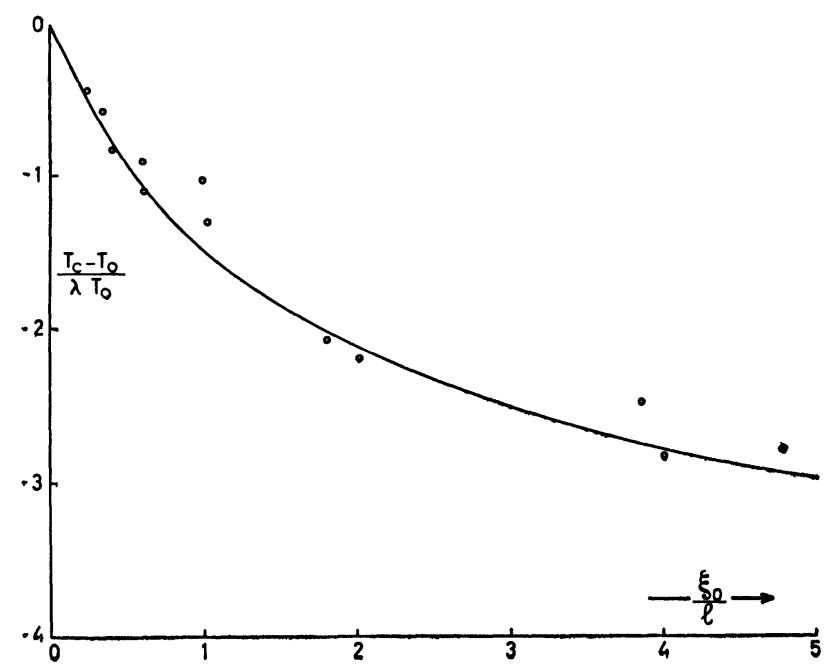

Fig. 1. - Variation de la température de transition $T_{\mathrm{o}}$ avec le rapport $\xi_{0} / l$ dans les alliages très dilués. En ordonrée on a porté $\left(T_{\mathrm{c}}-T_{0}\right) / T_{0} \lambda$ où $T_{0}$ est la température de transition du métal pur, et $\lambda$ un paramètre mesurant le taux d'anisotropie du gap dans le métal pur. Les points expérimentaux (réf. 2) sont relatifs aux alliages d'aluminium, avec des impuretés électropositives en faisant le choix $\lambda=0,13$. 
faiblement électropositives dans l'aluminium (*). On peut dans ce cas (sans faire de correction) reproduire avec une assez bonne précision la courbe empirique de Serin [2] en prenant $\lambda=0,13$ dans l'équation (II.25') (fig. 1). Les résultats de Serin suggèrent en outre que le taux d'anisotropie, mesuré par $\lambda$, est presque le même dans Al et dans Sn.

Dans le domaine des " alliages sales " $(A \ll B)$, l'opárateur " gap » $\Delta_{p}$ n'est pas strictement constant sur la snrface de Fermi, mais proportionnel à $F_{p}$ comme on le voit sur l'équation (II.16) (**). C'est l'amplitude de condensation ep paires $c_{p}=\left\langle a_{p} a_{-p}\right\rangle$ qui devient isotrope dans ce domaine. Le taux d'anisotropie de $c_{p}$ est proportionnel à

$$
\bar{K} \cong 1-N V\left[\psi_{1}\left(\frac{1}{2}-\frac{U_{0}}{2}\right)-\psi_{1}\left(\frac{1}{2}\right)\right] .
$$

III. Alliages «sales ». - 1 . ÉQUATION DE SELFCONSISTENCE POUR LE PARAMÈTRE D'ORDRE. Nous passons maintenant au domaine des alliages " sales » où le libre parcours moyen est très inférieur à la longueur de cohérence des constituants purs. Dans cette région les effets de l'anisotropie du gap sont devenus très faibles. Comme l'a remarqué Anderson [3] on peut alors, à une bonne approximation, décrire l'interaction électron-électron comme une interaction ponctuelle, mais retardée dans le temps. Nous allons rappeler tout d'abord les équations de base qui décrivent cette situation dans la méthode de Bogolubov. Aux opérateurs de création et d'annihilation $\psi+(r) \psi(r)$ nous substituons de nouveaux opérateurs $\gamma_{n}^{+} \gamma_{n}$ au moyen de la transformation linéaire .

$$
\begin{aligned}
& \psi \downarrow(r)=\sum_{n}\left(u_{n}(r) \gamma_{r} \uparrow+v^{\times}(r) \gamma_{n}^{+} \downarrow\right) \\
& \psi \downarrow(r)=\sum_{n}\left(u_{n}(r) \gamma_{1} \downarrow-v^{-} \times(r) \gamma_{n}^{+} \uparrow\right)
\end{aligned}
$$

Dans l'approximation de Hartree-Fock généralisée, les fonctions $u$ et $v$ sont les fonctions propres du système.

$$
\begin{gathered}
\varepsilon u(r)=\widehat{T} u(r)+\Delta(r) v(r) \\
-\varepsilon v(r)=\widehat{T} \quad v(r)-\Delta^{\times}(r) u(r) \\
\widehat{T}=-\frac{1}{2 m} \nabla^{2}+U(r)-E_{\mathrm{F}}
\end{gathered}
$$

représente l'énergie cinétique et l'énergie potentielle d'un électron dans l'approximation de

(*) L'origine de cette propriété est (en partie) expliquée dans la section III.

(**) Cette remarque a été faite par P. W. Anderson, L. Gruenberg et N. R. Werthamer.
Hartree, en présence des deux constituants de l'alliage. $\Delta(r)$ est le "potentiel de paires » défini par:

$$
\left.\Delta(r)=g(r)<\psi_{\uparrow}(r) \psi_{\psi}(r)\right\rangle .
$$

L'interaction attractive entre deux électrons situés en $r_{1}$ et $r_{2}$ est prise de la forme

$$
g\left(r_{1}\right) \delta_{3}\left(r_{1}-r_{2}\right)
$$

mais tronquée en fréquence : on suppose (en analogie avec l'apprnximation B. C. S.) que $\Delta(r)$ n'est non nul dans (III.2) que pour $\varepsilon<\omega_{D}$. On doit enfin écrire la condition de self consistence obtenue en injectant (1II.1) dans (III.3), et en écrivant que les états $\gamma_{n}^{+} d^{\prime}$ 'énergie $\varepsilon_{n}$, ont leur population d'équilibre thermique $f\left(\varepsilon_{n}\right)$. Ceci donne

$$
\Delta(r)=g(r) \sum_{n} v_{n}^{*}(r) u_{n}(r)\left(1-2 f\left(\varepsilon_{n}\right)\right) .
$$

Il y a aussi, en principe, un problème de self consistence pour déterminer le potentiel de Hartree qui figure dans $\widehat{T}(r)$, au voisinage d'une impureté par exemple [5]. En pratique toutefcis la distribution des charges n'est pratiquement pas affectée par la transition à l'état supraconducteur, et $\widehat{T}(r)$ peut donc être déterminé par une étude du métal normal, en s'aidant notamment de la règle de Friedel pour le cas d'impuretés diluées.

Par contre la construction d'une fonction $\Delta(r)$ self consistante dans un milieu non homogène pose en général un problème très difficile.

a) La méthode employée par Suhl [5] pour le cas des impuretés diluées revient à écrire la condition (III.4) en un point seulement (au centre de l'impureté). Il est difficile d'estimer sa précision.

b) La méthode utilisée par Anderson [3] et par Abrikosov et Gorkov [10] revient à remplacer dans (III.2) $\Delta(r)$ par sa moyenne spatiale. Dans ce cas les solutions $u_{n}(r), v_{n}(r)$ sont simplement proportionnelles aux fonctions d'onde $w_{n}(r)$ à un électron dans l'alliage normal, qui sont les fonctions propres de l'opérateur $\widehat{T}$. Physiquement, le remplacement de $\Delta(r)$ par sa moyenne est un peu analogue à l'approximation des bandes rigides telle qu'elle est utilisée par les alliages normaux. 11 peut être aussi décrit de la facon suivante : on connait les fonctions d'onde $w_{n}(r)$ dans l'alliage non supraconducteur. (En présence d'une impureté unique, par exemple, elles seraient de la forme.)

$$
w_{n}(r)=y_{l m}(\theta \varphi) f_{k}(r) \text {. }
$$

On construit une fonction d'essai pour décrire l'état supraconducteur en formant des paires à partir des états $w_{n}(r)$ et $\tilde{w}_{n}(r), \tilde{w}$ se déduisant de $w$ par renversement du sens du temps. Dans l'exemple ci-dessus $\tilde{w}=y_{l-m}(\theta \varphi) f_{k}$. On obtient ainsi l'approximation d'Anderson pour les supraconducteurs sales. Toutefois cette procédure n'est pas entièrement rigoureuse : on peut obtenir une éner- 
gie plus basse en formant des paires à partir d'états individuels qui sont un peu différents des $w_{n}(r)$. Nous verrons plus loin que cet effet est important, contrairement à ce qui est affirmé dans les références [3] et [10], lorsque l'on dissout dans un métal des impuretés de structure chimique très différente donnant lieu à des perturbations fortes.

e) Enfin, il existe un cas où l'on peut remplacer (III.4) par une condition de self consistence explicite et relativement simple : c'est le cas où $T$ est voisin de la température de transition $T_{\mathrm{c}}$ de l'alliage. Pour les alliages non magnétiques qui nous concernent seuls ici, la transition est toujours du $2^{\mathbf{e}}$ ordre et $\Delta(r)$ est très petit au voisinage de $T_{\mathbf{c}}$. On peut alors traiter $\Delta(r)$ comme une perturbation dans (III.2), et injecter les valeurs obtenues pour $u_{n}$ et $v_{n}$, du premier ordre en $\Delta$, dans (III.4). On obtient ainsi une équation de self consistence linéaire de la forme

$$
\Delta(r)=g(r) \int \mathrm{d}_{3} r^{\prime} K\left(r r^{\prime}\right) \Delta\left(r^{\prime}\right)
$$

avec

$$
\begin{gathered}
\boldsymbol{K}\left(\boldsymbol{r} \mathbf{r}^{\prime}\right)=\sum_{n m} \frac{\operatorname{th} \beta \xi_{n}}{\xi_{n}+\xi_{m}} \quad w_{n}(\boldsymbol{r}) w_{n}^{\times}\left(\boldsymbol{r}^{\prime}\right) w_{m}^{\times}(r) w_{m}\left(\boldsymbol{r}^{\prime}\right) \\
\left|\xi_{n}\right|<\omega_{\mathrm{D}} .
\end{gathered}
$$

Dans (III.6) nous avons convenu de prendre toutes les fonctions $w_{n}(r)$ comme réelles. Ceci est permis pour nos alliages non magnétiques, où l'hamiltonien à un électron $\widehat{T}$ est un opérateur réel. Notons encore que la quantité $1 /\left(\xi_{n}+\xi_{m}\right)$ doit être prise au sens d'une partie principale.

Les équations (III.5) et (III.6) sont l'équivalent spatial (pour une interaction ponctuelle) de l'équation (II.2). La forme (III.6) est souvent commode pour faire apparaître des règles de somme.

Par exemple si l'on fait la moyenne de (III.5) sur les configurations des impuretés, et si l'on suppose en première approximation que

$$
\overline{g(r) K\left(r r^{\prime}\right) \Delta\left(r^{\prime}\right)} \rightarrow \overline{g(r) K\left(r r^{\prime}\right)} \bar{\Delta}
$$

(ce qui est essentiellement l'approximation d'Anderson [3]),

On peut facilement calculer $\int \overline{\left.K r r^{\prime}\right)} d_{3} r^{\prime}$ en utilisant les propriétés d'orthogonalité des fonctions $w_{n}(r)$. On obtient

$$
\begin{aligned}
\int K\left(r r^{\prime}\right) d_{3} r^{\prime} & =N(r) \log \frac{1,14 \omega_{D}}{T} \\
\int \overline{g(r) K\left(r r^{\prime}\right) d_{3} r^{\prime}} & =\overline{g N} \log \frac{1,14 \omega_{D}}{T}
\end{aligned}
$$

où $N(r)=\sum_{n} \mid w_{n}(r)^{2} \delta\left(\xi_{n}\right)$ est la densité locale de niveaux par $\mathrm{cm}^{3}$ et par unité d'énergie. On tire de (III.9) et (III.5) une température de transition.

$$
T_{0}=1,14 \omega_{D} \mathrm{e}^{-\frac{1}{\sigma N}}
$$

On peut aussi, en faisant des approximations analogues à (III.7) calculer la longueur de cohérence et l'épaisseur de pénétration d'un champ magnétique dans les alliages concentrés en fonction de la conductivité. Nous réservons toutefois cette question pour un article ultérieur.

2. Distorsions locales de paramètre D'ORDRE $\Delta(r)$. - Nous allons maintenant discuter plus en détail la validité de l'approximation (III.7) sur un cas particulièrement schématique, mais tout de même instructif : il s'agit du cas où l'interaction entre électrons n'est pas affectée par les impuretés : $g(r)=g=$ constante.

Dans ce cas les calculs sont particulièrement simples. Ils montrent que lorsque les deux constituants de l'alliage sont très différents chimiquement, l'approximation (III.7) est en fait très insuffisante.

Pour résoudre (III.5), nous utilisonsune méthode d'itération.

Dans l'approximation d'ordre zéro nous prenons $\Delta(r)=1$.

En utilisant l'orthogonalité des fonctions $w_{n}$ on peut calculer explicitement le résultat de la première itération.

$$
\begin{gathered}
\Delta^{(1)}(r)=\bar{g} \sum_{n}\left|w_{n}(r)\right|^{2} \frac{\operatorname{Th}\left(\frac{\beta \xi_{n}}{2}\right)}{2 \xi_{n}} \\
\left|\xi_{n}\right|<\omega_{D} .
\end{gathered}
$$

Définissons toujours la densité locale de niveaux par

$$
N(\boldsymbol{r})=\sum_{n}\left|w_{n}(r)\right|^{2} \delta\left(\xi_{n}\right) .
$$

Nous obtenons après intégration sur les énergies

$$
\Delta^{(1)}(r)=\log \frac{1,14 \omega_{D}}{T} N(r)
$$

c'est-à-dire un paramètre d'ordre $\Delta(r)$ proportionnel à $N(r)$, ce résultat constitue un progrès sensible sur l'approximation (III.7)

Pour voir comment il affecte la température de transition, écrivons la deuxième itération

$$
\Delta^{(2)}(r)=\bar{g} \log \frac{1,14 \omega_{D}}{T} \int K\left(r r^{\prime}\right) N\left(r^{\prime}\right) \mathrm{d}_{3} r^{\prime}
$$

Il n'est pas possible d'écrire explicitement $\Delta^{2}(r)$. Toutefois, nous pouvons obtenir la condition qui définit la température de transition en égalant les valeurs moyennes $\overline{\Delta^{(1)}(r)}$ et $\overline{\Delta^{(2)}(r)}$ obtenues par les deux dernières itérations. En effet $\overline{\Delta^{(2)}(r)}$ peut être calculé explciitement en utilisant encore l'orthogonalité des $w_{n}$. On obtient

$$
\begin{gathered}
\overline{N(r)}=g \sum_{n} \int \mid w_{n}\left(r^{\prime}\right)^{2} N\left(r^{\prime}\right) d_{3} r^{\prime} \frac{T h\left(\frac{\beta \xi_{n}}{2}\right)}{2} \\
\left|\xi_{n}\right|<\omega_{D} \\
\overline{N(r)}=g \log \frac{1,14 \omega_{D} \overline{N^{2}(r)}}{T_{\mathrm{c}}} \\
T_{\mathrm{c}}=1,14 \omega_{D} \mathrm{e}^{-\frac{1}{N_{\text {effg }}}} \\
N_{\text {eff }}=\overline{N^{2}} / \bar{N}
\end{gathered}
$$


Il est intéressant de voir la forme que prend ce résultat pour des impuretés dilués. Écrivons

$$
N(r)=N_{0}+\delta N
$$

où $N_{0}$ est la densité de niveaux dans la matrice, et $\delta N$ la modification présente au voisinage des impuretés ; $\delta N / N_{0}$ n'est pas nécessairement petit. mais $\overline{\delta N} / N_{0}$ est petit (simplenent parce que les impuretés sont diluées).

On a dans ce cas

$$
\begin{aligned}
N_{\text {eff }}=\frac{\overline{N^{2}}}{\bar{N}} & =\frac{N_{0}^{2}+2 N_{0} \overline{\delta N}+\overline{\delta N^{2}}}{N_{0}+\overline{\delta N}} \\
& =N_{0}+\overline{\delta N}+\frac{\overline{\delta N^{2}}}{N_{0}} \\
& =N_{0}+\overline{N \delta N} / N_{0} .
\end{aligned}
$$

Pour des impuretés diluées (en concentration $n$ ) le terme en $\overline{N \delta N}$ est la somme de contributions indépendantes des différentes impuretés, et l'on obtient

$N_{\text {eff }}=N_{0}+n \int \frac{N(r)\left(N(r)-N_{0}\right)}{N_{0}} \mathrm{~d}_{3} r$

Dans (III.17) l'intégrale est étendue au voisinage d'une impureté unique dans la matrice pure.

Il est intéressant de comparer les températures de transition $T_{\mathrm{c}_{1}}$ calculées par la première approximation (éq. III.10) avec celles $T_{\mathrm{c}_{2}}$ déduites de (III.15) et (III.17). Nous avons fait ce calcul pour différentes impuretés dans des matrices d'aluminium et d'étain. La méthode adoptée consiste à décrire la matrice comme un gaz d'électrons libres, et l'impureté comme un puits carré de potentiel, occupant une sphère égale au volume atomique, et dont la profondeur est ajustée pour satisfaire à la règle de Friedel [11]. (Les calculs ont été faits en utilisant 4 déphasages.) On calcule les fonctions d'onde, puis la densité $N(r)$, les quantités $\bar{N}$ et $N_{\text {eff }}$. On a inclus uniquement les effets de densité de niveau ; c'est-à-dire que $g$ est pris égal à sa valeur dans la matrice pure.

Finalement on calcule le quotient :

$$
S=\frac{T_{\mathrm{c}}-T_{0}}{n v_{0}}
$$

ou $v_{0}$ est le volume atomique et $n v_{0}$ le pourcentage d'impuretés. Le ta.bleau 1 donne les résultats dans la première approximation $\left(S_{1}\right)$ et dans la deuxième $\left(S_{2}\right)$ pour différents excès de charge $Z$ apportés par l'impureté.

Les principales conclusions sont les suivantes :

a) On vérifie que $S_{2}$ est toujours plus grand que $S_{1}$ ( $N_{\text {eff }}>\bar{N}$; comme on pouvait le prévoir d'après la définition de $N_{\text {etr }}$ ) dans la deuxième approximation, on permet aux paires de se former à partir d'états plus favorables que ceux

$$
\left(\mathfrak{w}_{n}(r) \tilde{w}_{n}(r)\right)
$$

utilisés dans la première approximation.

\section{TABLEAU 1}

Effet de la densité de niveaux SUR LE POINT DE TRANSITION

Impuretés dans $\operatorname{Sn}\left(T_{0}=3,75, N_{0} g=0,25\right)$

$$
\begin{array}{cccccc} 
& Z=1 & -1 & -2 & -3 & -4 \\
S_{1} & \overline{2,19} & -\overline{1,56} & -\overline{4,46} & -\overline{7,59} & -\overline{11,3} \\
S_{2} & 2,40 & -1,49 & -3,72 & -5,12 & -6,91
\end{array}
$$

Impuretés dans $\mathrm{Al}\left(T_{0}=1,2{ }^{\circ} \mathrm{K}, N_{0} g=0,17\right)$

$$
\begin{array}{cccccc} 
& Z=2 & 1 & -2 & -2 & -3 \\
S_{1} & \overline{1,30} & \overline{0,71} & -\overline{0,85} & -\overline{1,45} & -3,57 \\
S_{2} & 1,51 & 0,80 & -0,73 & -0,62 & -1,04
\end{array}
$$

b) $S_{2}$ est peu différent de $S_{1}$ pour $Z= \pm 1$. Par contre, pour $|Z|$ élevé, les résultats sont considérablement modifiés : l'effet des distorsions locales du paramètre d'ordre $\Delta(r)$ est important.

Il n'est pas possible de comparer directement $S_{2}$ aux valeurs expérimentales (mesurées dans le domaine des alliages "sales " $\left(1 / \tau \ll T_{\mathrm{c}}\right)$ mais encore dilués $\left.\left(n v_{0} \ll 1\right)\right)$. Il faudrait tenir compte en effet des variations de $g$ et des modifications du spectre de phonons dans l'alliage. Le terme dominant, toutefois, est bien donné par $S_{2}$ et son signe, en particulier, est en accord avec l'expérience pour l'énorme majorité des alliages $\mathrm{Sn}$ et $\mathrm{Al}$ mesurés jusqu'à présent [1], [2].

3. Distorsion De $\Delta(r)$ AU voisinage D'Une IMPURETÉ UNIQUE OU D'UNE SURFAGE LIBRE. - Il est assez intéressant d'étudier en détail l'aspect de $\Delta(r)$ au voisinage d'une impureté unique dans une matrice supraconductrice, en se plaçant par exemple exactement à la température de transition $T_{0}$ de celle-ci. Les calculs peuvent être conduits en utilisant les méthodes d'itération présentées ci-dessus. Les résultats essentiels sont les suivants :

a) au voisinage de l'impureté $\Delta(r)$ est essentiellement proportionnel à $g(r) N(r)$ et varie donc violemment ;

b) aux très grandes distances $\left(r>\xi_{0}\right)$ l'équation macroscopique de Landau-Ginsburg [12], [6], s'applique. Elle peut être écrite sous la forme

$$
\Delta(r)\left(\frac{T-T_{0}}{T_{0}}\right)=L \nabla^{2} \Delta(r)+0\left(\Delta^{2}\right) .
$$

Pour $T=T_{0}$, où l'on peut supposer $\Delta$ très faible, elle donne $\nabla^{2} \Delta(r)=0$

$$
\Delta(r)=\Delta_{0}\left(1+\frac{\alpha}{r}\right)
$$

$\Delta_{0}$ est la valeur (très petite) du paramètre d'ordre dans la matrice, $\alpha$ est une longueur, que nous 
appellerons l'amplitude de diffusion de $\Delta$. Dans une approximation équivalente à (III.15) on trouve :

$$
\alpha=\frac{1}{4 \pi L} \int \frac{\left(N(r)-N_{0}\right) N(r)}{N_{0}^{2}} \mathrm{~d}_{3} r .
$$

Le coefficient $L$ relatif à la matrice pure, est de l'ordre de $\xi_{0}^{2}$. L'intégrale $\int d_{\mathbf{8}} r$ dans (III.30) est au plus de l'ordre de $a^{3}$, ou $a$ est le rayon de la charge déplacée. par l'impureté (comparable à une distance interatomique). Donc $\alpha \sim a^{3} / \xi_{0}^{2}$. Dans un matériau doux $a / \xi_{0} \sim 10^{-3}$ et $\alpha \sim 10^{-6} a$ est une longueur extraordinairement faible. Toutefois l'effet décrit par (III.20) n'est pas négligeable dès que l'on a une concentration finie d'impuretés (chaque impureté contribuant à de très grandes distances). C'est lui qui est en fait responsable du déplacement $T_{0} \rightarrow T_{0}$, et on peut d'ailleurs retrouver une équation équivalente à (III.15) aux faibles concentrations, en appliquant le " théorème optique " à (III.20).

Notons en passant que l'ordre de grandeur de l'amplitude $\alpha$ serait complètement différent si nous avions affaire à une impureté magnétique. On peut montrer en effet que dans ce cas $\alpha$ est de l'ordre de $a^{2} / \xi_{0}$, donc énormément plus grand que dans le cas précédent.

Enfin, on peut appliquer les mêmes méthodes à l'étude de $\Delta(r)$ au voisinage de la surface libre d'un métal pur : ceci permet de rendre explicite la condition aux limites qui doit être appliquée à l'équation macroscopique de Landau-Ginsburg [12] au voisinage d'une telle surface.

Il est nécessaire (mais non suffisant) d'imposer une condition assurant que le courant normal à la surface est nul. Dans la jauge de London, par exemple, cette condition est automatiquement satisfaite si $\Delta$ est réel. Il reste encore à préciser dans ce cas la pente de $\Delta$ à la surface. Expérimentalement, on sait que le point de transition de spécimens dont l'épaisseur est comparable à $\xi_{0}$ est le même que celui de spécimens massifs, et ceci montre que la condition aux limites correcte correspond pratiquement à une pente nulle. Ceci ne semble ‘pas, toutefois, avoir été justifié jusqu'ici par un calcul détaillé. Nous allons donner ici les grandes lignes du calcul, avec les mêmes approximations que dans le paragraphe 2 .

La surface $S$ du métal est supposée plane (plan x Oy . Le supraconducteur occupe la région $z>0$ et $\Delta(r)$ dépend seulement de $z$ (fig. 2). Nous nous efforçons toujours de résoudre (III.5 (avec $g$ constant pour simplifier). Dans le cas présent les fonctions $\mathfrak{W}_{n}(r)$ et la densité de niveaux $N(r)$ s'annulent exponentiellement pour $z<0$. Si nous prenons encore comme approximation d'ordre zéro un gap constant $\Delta^{\circ}$ nous obtenons par une première iłération le résultat (III.13). Plaçons nous à la température de transition $T_{0}$ du métal massif.
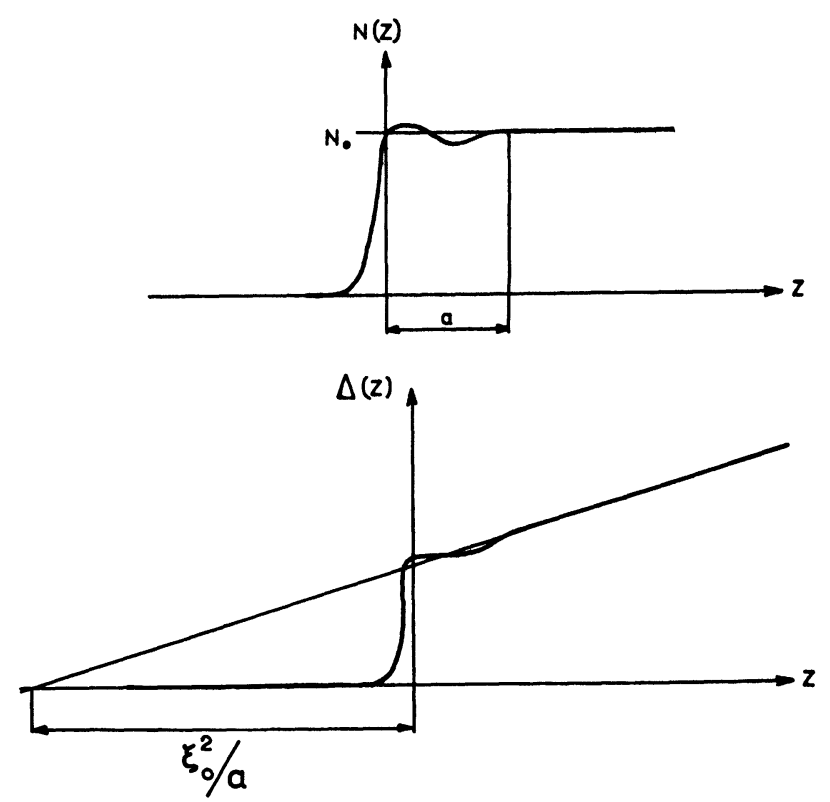

Frg. 2. - Variation de la densité locale de niveaux au voisinage de la surface du métal ( $f$ g. $2 a$ ) et variation résultante du paramètre d'ordre $\Delta$. Par nécessité la figure est dessinée à une échelle très incorrecte. En fait la longueur $\xi_{0}^{2} / a$ est énorme par rapport à $a$.

Ceci permet d'écrire $\Delta^{(1)}(r)=\frac{N(r)}{N_{0}} \Delta^{0}$. Dans cette premiçre approximation le paramètre d'ordre s'annule dans la région vide, puis retourne à une valeur constante $\Delta^{(0)}$ dès que $z$ est plus grand que quelques distances interatomiques. Ceci ne correspond pas, toutefois, à la solution la plus générale de l'équation macroscopique (III.19). En effet pour $T=T_{0}$, celle-ci nous donne

$$
L \frac{\mathrm{d}^{2} \Delta}{\mathrm{d} z^{2}}=0
$$

c'est-à-dire que $\Delta$ est en général une fonction linéaire de $z$ dans la région intérieure. Le problème est précisément de déterminer la pente de $\Delta$ mesurée loin de la surface $(\mathrm{d} \Delta / \mathrm{d} z)_{z>\varepsilon_{0}}$. On y parvient de la façon suivante :

Soit $K^{\circ}\left(r r^{\prime}\right)$ le noyau correspondant à (III.6) pour le cas d'un métal occupant tout l'éspace $(z>0$ et $z<0)$ [ $K^{\circ}$ ne dépend que de $\left.\left(r-r^{\prime}\right)\right]$.

Posons $\delta K\left(r r^{\prime}\right)=K\left(r r^{\prime}\right)-K^{\circ}\left(r r^{\prime}\right)$ et écrivons l'équation (III.5) sous la forme

$$
\begin{aligned}
& \Delta(r)-g \int K^{0}\left(r r^{\prime}\right) d_{3} r^{\prime} \Delta\left(r^{\prime}\right) \\
& =g \int \delta K\left(r r^{\prime}\right) \Delta\left(r^{\prime}\right) d_{3} r^{\prime}=H(r) .
\end{aligned}
$$

Dans le nombre de droite de (III.22) nous injecterons la valeur approchée (III.21) du gap $\Delta^{(i)}(r)$. La fonction $H(r)$ n'est non nulle que dans une région d'épaisseur $\sim \xi_{0}$ au voisinage de la surface. 
Nous allons calculer de deux façons l'intégrale $\int \mathrm{d}^{3} r H(r)$. En utilisant la deuxième égalité de (III.22) et les propriétés $K\left(r r^{\prime}\right)$ on obtient :

$\int H(r) \mathrm{d}_{3} r=g \int \mathrm{d}_{3} r^{\prime}\left(N\left(r^{\prime}\right)-N_{0}\right) \frac{N\left(r^{\prime}\right)}{N_{0}} \Delta^{0}=S a \Delta^{0}$

où $S$ est la surface du spécimen, et $a$ une longueur qui donne en gros l'épaisseur dans laquelle $N(r)$ passe de la valeur 0 à la valeur $N_{0}$ ( $a$ est donc de l'ordre d'une distance interatomique). D'autre part, en utilisant la première égalité (III.22) on obtient

$$
\begin{aligned}
& \int_{-\infty}^{z_{0}} \mathrm{~d} z \int \mathrm{d} x \mathrm{~d} y \boldsymbol{H}(\boldsymbol{r}) \\
& =\int_{-\infty}^{z_{0}} \mathrm{~d} z \int \mathrm{d} x \mathrm{~d} y\left[\Delta(\boldsymbol{r})-g \int K^{\mathrm{o}}\left(\boldsymbol{r} \boldsymbol{r}^{\prime}\right) \Delta\left(\boldsymbol{r}^{\prime}\right) \mathrm{d}_{3} \boldsymbol{r}^{\prime}\right] \\
& =g \int_{-\infty}^{z_{0}} \mathrm{~d} z \int \mathrm{d} x \mathrm{~d} y \mathrm{~d}_{3} \boldsymbol{r}^{\prime}\left(\Delta(\boldsymbol{r})-\Delta\left(\boldsymbol{r}^{\prime}\right)\right) K^{\mathrm{o}}\left(\boldsymbol{r} \boldsymbol{r}^{\prime}\right) \quad \text { (III.24) } \\
& =g \int_{-\infty}^{z_{0}} \mathrm{~d} z \int_{z_{0}}^{\infty} \mathrm{d} z^{\prime} \mathrm{d} x \mathrm{~d} y \mathrm{~d} x^{\prime} \mathrm{d} y^{\prime}\left(\Delta(\boldsymbol{r})-\Delta\left(\boldsymbol{r}^{\prime}\right)\right) K^{\circ}\left(\boldsymbol{r} \boldsymbol{r}^{\prime}\right)
\end{aligned}
$$

choisissons $z_{0}>\xi_{0}$. Alors les équations macroscopiques s'appliquent et nous pouvons écrire :

$$
\Delta(r)-\Delta\left(r^{\prime}\right)=\frac{\mathrm{d} \Delta}{\mathrm{d} z}\left(z-z^{\prime}\right)
$$

où $\mathrm{d} \Delta / \mathrm{d} z$ est une constante (la pente «macroscopique " de $\Delta$ ). On peut alors effectuer l'intégration $\int \mathrm{d} r$ dans (III.24) et on obtient

$$
\int \mathrm{d}_{3} r H(r)=S L \frac{\mathrm{d} \Delta}{\mathrm{d} z} \text {. }
$$

En comparant (III.26) et (III.23) nous trouvons la condition aux limites, macroscopique reliant la valeur et la pente de $\Delta$ à la surface

$$
\frac{\Delta_{0}}{\frac{\mathrm{d} \Delta}{\mathrm{d} z}}=\frac{L}{a} .
$$

La longueur $L / a$ est de l'ordre de $\xi_{0}^{2} / a$, donc très grande. En pratique la condition $\mathrm{d} \Delta / \mathrm{d} z=0$ est donc approximativement correcte. En particulier, dans les matériaux doux où $\xi_{0}$ est élevé, on calcule correctement l'effet du champ sur l'épaisseur de pénétration en utilisant la condition aux limites $\mathrm{d} \Delta / \mathrm{d} z=0$.

Les résultats de cette section ont été établis pour le cas particulier d'une interaction ponctuelle iso- trope $g \delta_{3}(r)$. Il est facile de voir qu'ils restent qualitativement inchangés si l'on utilise une interaction $g\left(r-r^{\prime}\right)$ plus générale, mais que l'on néglige toujours les effets d'anisotropie. En particulier on peut encore définir le cas d'une amplitude $\alpha$, par l'équation (III.20), pour une impureté isolée dans une matrice cubique, à partir des équations macroscopiques de Landau-Ginsburg, et l'ordre de grandeur de $\alpha$ est toujours $a^{3} / \xi_{0}^{\prime}$. Il serait intéressant d'étudier comment sont modifiés ces résultats, quand on inclut l'anisotropie de la surface de Fermi et des interactions, de façon à établir un lien entre cette description et celle de la section II pour les alliages très dilués.

IV. Conclusions. - Au terme de cette étude il apparait que nous sommes actuellement en mesure de tenir compte assez correctement des effets liés à la diffusion des électrons par les impuretés, dans le domaine très dilué où la forme détaillée de l'interaction électron-électron doit être prise en compte.

Dans le domaine des alliages "sales " pour avoir des résultats maniables, nous avons dû utiliser une interaction électron-électron ponctuelle. En fait, la portée spatiale de l'interaction électron phonon électron est au moins de l'ordre du rayon d'écran dans le métal.

La perturbation due à une impureté localisée a donc un rayon comparable, et on ne peut pas espérer qu'une théorie fondée sur une interaction ponctuelle donne des résultats très quantitatifs.

En outre, nous avons supposé dans la plupart des applications que cette interaction n'est pas modifiée par l'impureté, ce qui est inexact (mais empiriquement assez satisfaisant).

Nous nous proposons de revenir ultérieurement sur ces problèmes, ainsi que sur les propriétés électrodynamiques des alliages sales.

Remerciements. - Nous remercions tout particulièrement MM. J. Friedel, H. Suhl et P. W. Anderson, pour plusieurs discussions reliées aux alliages supraconducteurs, et le Dr E. Abrahams, qui a bien voulu nous communiquer le texte non publié de sa contribution à la conférence de Cambridge, 1959. Une partie de ce travail a été effectuée alo's que l'un de nous (P. G. de G.) bénéficiait de l'hospitalité de l'Institut de Physique Théorique de l'Université de Kyoto. Il tient à remercier notamment le Dr Mori et le Dr Tsuneto.

\section{REFERENCES}

[1] Lynton (E.), Serin (B.) et Zucker, J. Phys. Chem. Solids, 1957, 3, 165 .

Chanin, Lynton (E.) et Serin (B.), Phys. Reo., 1959, 114, 719.

[2] SERIN (B.), Proceedings of the Toronto conference on low temperature physics, 1960, p. 391.

Lynton et MacLean, Phys. Rev., 1962, 126, 40.
[3] Anderson (P. W.), J. Phys. Chem. Solids, 1959, 11, 26.

ANDERson (P. W.), Proceedings of the Toronto conference on low temperature physics, 1960, p. 298.

[4] Abrahams (E.) et Weiss (P. R.), Communication à la conférence de Cambridge, 1959. 
[5] Suml (H.), Low temperature physics, cours de l'École des Houches, 1961 (Gordon et Breach, New-York), p. 321.

[6] Bardeen (J.), Cooper (L. N.) et Schrieffer (S.), Phys. Res., 1957, 108, 1175.

[7] Gon'kov (L. P.), J. Exp. Theor. Phys., U. R. S. S., 1959, 36, 1918 ; Soviet Physics (J. E. T. P.), 1959, 9,1364 .

[8] Pour une revue de ces méthodes, dues essentiellement à Edwards (S. F.), Abrikosov (A.) et Gor'kov (L. P.), voir par exemple Rickayzen, cours de l'école d'été de Bergen, 1960.
[9] Hebel (L. C.) et Slichter (C. P.), Phys. Res., 1957, 107, 401 et Phys. Rev., 1959, 113, 1503. RED Field (A. G.), Phys. Rev. Letters, 1959, 3, 85. Pour une mise au point récente sur l'aluminium, voir MASUdA (Y.), Phys. Reo., 1962, 126, 1271.

[10] Abrikosov (A.) et Gor'kov (L. P.), J. E. T. P., U. R. S. S., 19t9, 36, $319 ; 1960,39,1781$.

[11] Friedel (J.), Ado. in Physics, 1954, 3, 446.

[12] Ginsburg (V. L.) et Landau (L. D.), J. E. T. P., U. R. S. S., 1959. 20, 1064. 